\title{
The Definiteness Effect in Attributive Comparatives
}

\author{
Franz Beil \\ IMS, Universität Stuttgart
}

\section{Introduction}

The aim of this paper is to give an explanation for the definiteness effect (DE) that arises with attributive comparative constructions. The DE in comparatives secms to have been noticed only as recently as in (Lerner and Pinkal 1995). For an illustration, consider the contrast between examples (1) and (2). While (1) is fine, (2) is at least odd if not ungrammatical.

(1) $\mathrm{Al}$ ate a bigger piece of cake than Sue (did).

(2) \# Al ate every bigger piece of cake than Sue (did).

As with other environments that exhibit a definiteness effect or a definiteness restriction, as for instance there be sentences, cleft or pseudocleft construction etc. (cf. Higginbotham (1987) for a list), the DE in attributive comparatives arises with all types of strong comparative NPs, i.e. comparative NPs that contain a strong determiner, e.g. a universal determiner, a definitc article, a partitive determiner etc.

$$
\text { \# Al ate }\left\{\begin{array}{c}
\text { most } \\
\text { the } \\
\text { two of the }
\end{array}\right\} \text { bigger piece(s) of cake than Sue (did). }
$$

In contrast, comparative NPS with a weak determincr as an indefinite article or determiner, a (modified) numerical determiner etc. are fine.

$$
\mathrm{Al} \text { ate }\left\{\begin{array}{c}
\text { a few } / \text { many } \\
\text { some } \\
\text { (exactly) two } \\
\text { at most/least two }
\end{array}\right\} \text { bigger pieces of cake than Sue (did). }
$$

Lerner and Pinkal (1995) claim that the DE is dependent on the explicit occurrence of a comparative complement. They motivate their claim with examples as the following.

(5) George owns every/the faster car.

(their (32) and (33))

Note, however: that although the DE appears to be dependent on an explicit occurrence of a comparative complement, this does not mean that the presence of a complement is a sufficient condition for the DE. Consider the following example cluster with strong comparative NPs and a complement:

(6) $\mathrm{Al}$ ate every/the/most bigger piece(s) of cake than this one.

(C) 1997 by Franz Beil

Aaron Lawson (ed), SALT VII, 37-54, Ithaca, NY: Cornell University. 
Moreover, it is disputable whether the complementless comparative does not show the DE in general. Although a comparative that is lacking a complement does not give a comparison instance ${ }^{1}$ explicitly, it is nonetheless required for the interpretation of the comparative clause. For both weak and strong comparative NPs that lack a complement, some comparison instance has to be provided for in the context. On this view, one may suspect that the particular way in which an instance is contextually given may have an effect similar to the DE on the contextual coherence of a comparative sentence. This is witnessed by the differences in acceptability of the following sentence pairs.

(7) Of those cars, Sue bought one. George bought every faster car.

(8) Sue bought a car. \#George bought every faster car.

(9) Sue bought a car. George bought a faster car.

Intuitively, the difference between (7) and (8) is that, in (7), a distinguished clement in a given set of instances, namely the instance given by Sue's car, facilitates to determine the denotation of every faster car on the basis of the given set of cars. Contrastingly, the first sentence in (8) only renders available some comparison instance without a definite set from which it is taken. The lack of a contextually given set of comparison instances doesn't seem to matter in (9), where the comparative NP is weak.

The structure of the paper is as follows. In section 2., I argue that comparative adjectives have particular contextual properties which show certain similarities to presuppositions. The notion of a comparison set is introduced and motivated. Section 3. reviews a promising approach to the DE in general by Moltmann (1996). An analysis of the differences between weak and strong NPs is given in terms of domain presupposition. Section 4. and 5. provide a proposal for the logical form of the comparative NP and the complement. Section 6. gives an account of the DE in attributive comparatives on the basis of the analysis in sections 4 . and 5. Finally, section 7. adds an outline of my view about the resolution of various forms of comparative ellipsis.

\section{Presupposition-like properties of comparatives}

Before attempting a formalization of the semantics of attributive comparative constructions, I motivate the above claim that the presence of a comparison instance is a contextual requirement for the interpretation of comparatives. Consider the scapegoat assumption that the comparison instance is scmantically part of the assertion of a comparative. Then, one might expect a comparative without complement to pattern like constructions that are assumed to have implicit arguments that can be inferred on the basis of their function's specification. Take for instance the intransitive Sue ate, where an unspecified theme, though missing, is an implicit part of the assertion and, 
therefore, can be inferred: there has to be something Sue ate when it is the case that she ate. Since implicit arguments are constituting parts of the assertion, the inferences that can be drawn on the basis of such an argument depends on the assertion as a whole. If, for example, an assertion has the form of a negation, the inferences drawn on the basis of an implicit argument are not the same as those of the corresponding positive asscrtion. Sue didn't eat does not entail that there is something Sue did not eat. Returning to the complementless comparative, we observe that the information that there has to be an implicit comparison instance survives particular properties of the assertion. The following examples are examples for the common presupposition tests of negation and counterfactual conditionals respectively.

(10) Sue didn't eat a bigger piece of cake.

(11) If Sue ate a bigger piece of cake, she would be contrite.

For an assertion of (10) to be felicitous, there has to be some piece of cake or, more cautiously, some somehow sized object that serves as the comparison instance. Likewise for (11). Thus, the instance obeys the presupposition tests. Further, if the comparative complement is a means to express such an instance, its interpretation should be immune to the particular environment it occurs in. This means we do not expect any effect on the comparison instance when a comparative NP with complement occurs in a negation or in a conditional. Since, for reasons of possible scope interactions, conditionalizing is the more reliable presupposition test, we confine ourselves to the following.

(12) If Sue ate a bigger piece of cake than $\mathrm{Al}$, she would be contrite.

Here, the contextually required instance is in some way related to Al. Neither the requirement for such an instance nor its being related to $\mathrm{Al}$ is affected by the fact that the comparative clause occurs in the antecedent of the conditional.

Another hypothesis about the status of the complement is that it modifies the comparative noun or NP, i.e. it is semantically a function on $\mathrm{N}$ or NP denotations comparable, for instance, to relative clauses or NP modifying PPs. Again, counterevidence can be drawn from their behaviour in presupposition tests. The information of modifiers like PPs or relative clauses does not survive in conditionals. For (13) and (14), it is not required that Al made a cake and that there is a coconut cake, respectively.

(13) If Sue would eat a cake Al made, she wouldn't like it.

(14) If Sue would eat a cake with coco flakes, she would have an allergic fit.

As has been claimed in the introduction, I take the above considerations as evidence that the presence of a complement is a means to identify a subset in a possibly bigger set of contextually available comparison instances rather than to assert the instance itself. Before giving an explication of this I introduce a promising analysis of the DE in general and examine the DE in comparatives more closely. 


\section{The definiteness effect as a discourse-related phenomenon}

The definiteness effect in there be sentences received by far the most attention in the literature. Apart from the problem of the particular semantics of there be, the main question concerned the charactcristics of the NPs acceptable in there be constructions.

(15) There is a/at least one/no sparrow on the roof.

(16) \# There is every/the sparrow on the roof.

The examination of the logical properties of determiners in NPs provided the means for a descriptive generalization: NPs acceptable in there be constructions have a determiner that is intersective. In order to determine the denotation of a NP containing an intersective determiner one only needs to know the intersection of its two arguments. We take the respective definition from Keenan (1996) (for the further, call $D$ 's first argument the domain of $D$ and the second argument its scope):

Df 1. A determiner $D$ is intersective iff for any sets $A, B, A^{\prime}, B^{\prime} \subseteq U$ :

$$
A \cap B=A^{\prime} \cap B^{\prime} \Rightarrow D(A)(B)=D\left(A^{\prime}\right)\left(B^{\prime}\right)
$$

Another problem concerns the explanation of why only NPs with intersective determiners and not others are subject to the DE. A promising approach to such an explanation is given in (Moltmann 1996), who rather focusses on the characteristics of NPs not acceptable in constructions that give rise to a DE. Before giving a synopsis of Moltmann's arguments, let me add some motivation from the logical characteristics of NPs subject to a DE. As regards unacceptable, simple NPs, i.c. NPs containing just a lexical determiner, the relevant property of the determiner seems to be what has been classified as co-intersectivity:

Df 2. A determiner $D$ is co-intersective iff for any sets $A, B, A^{\prime}, B^{\prime} \subseteq U$ :

$$
A-B=A^{\prime}-B^{\prime} \Rightarrow D(A)(B)=D\left(A^{\prime}\right)\left(B^{\prime}\right)
$$

Drawing from the particular set-theoretic operation used in the definitions of intersective and co-intersective determiners respectively, an important minimal difference can be shown with conservativity of $D$ :

Thm 1. All intersective determiners $D$ are symmetric:

$$
\begin{array}{lr}
\text { for any } A, B \subseteq U: D(A)(B)=D(B)(A) & \text { from Df } 1 \\
\Rightarrow \quad D(A)(B)=D(A \cap B)(A \cap B) & \text { with conservativity }
\end{array}
$$

Thm 2. Co-intersective determiners $D$ are not symmetric (for $|U| \geq 1$ ):

$$
\begin{array}{lr}
\text { there } \operatorname{arc} A, B \subseteq U: D(A)(B) \neq D(B)(A) & \text { from Df } 2 \\
\Rightarrow \quad D(A)(B)=D(A)(A \cap B) & \text { with conservativity }
\end{array}
$$

with conservativity

The respective bottom lines show the familiar fact that co-intersective (strong) determiners are dependent on their domain's definition while intersective 
(weak) determiners are not. For a NP with a co-intersective determiner, one has to know what $A$ is in order to determine the denotation of the NP. Now, Moltmann claims that this logical property of co-intersective simple determiners is a reflex of a general contextual requirement for non-intersective quantifiers $^{2}$ :

(DP) Domain Presupposition Thesis

(Moltmann 1996, p.4):

Non-intersective (strong) quantifiers always presuppose their domain.

Crucially, domain presupposition is not only that the domain of a strong quantifier be non-empty but that the domain set is given by the previous context. Preeminent support for the claim that strong quantifiers carry a domain presupposition comes from the analysis of NPs that contain event-dependent modifiers such as new, other or first. A further property of those modifiers, somewhat neglected in (Moltmann 1996), is that they assert a property of an individual on the background of a contextually given comparison class: in order to characterize something as new, other or first, one has to determine with respect to what comparison class it is so characterized. I account for this fact by the following logical form for the respective modifiers:

(17) new $\Longrightarrow \lambda P \lambda x P(x) \wedge n w^{\prime}(x, \mathbf{X})$

$P$ is the property to be determined by the noun; the comparison class is a set $\mathrm{X}$ that has to be contextually determined (one might add a constraint that $\mathrm{X} \subseteq P$ ).

In this light, of particular interest are the readings in which the determination of the comparison set is in some way dependent on the event described by the verb of the clause the modifier occurs in. Consider the following contrast in available readings for a sentence containing a weak NP the domain of which is modified by new in (18), and onc with a respectively modified strong NP in (19).

(18) John used a/at least one/no new pen.

(Moltmann's (5a))

a. John used a pen he recently acquired

b. John used a pen different from the ones he used before.

(19) John used every/the new pen.

(Moltmann's (10))

a. John used every pen he recently acquired.

b. \#.John used every pen different from the ones he used before.

Moltmann describes the contrast as follows. (18) is acceptable on a reading that involves a comparison class to be determined relative to "an event of the same type as [the event described by the sentence]", while (19), with a strong determiner with a respectively modified domain, is not. It is an intricate problem what "an event of the same type" is supposed to mean here. Let me suppress the discussion the avalanche of problems for its exact characterization 
and assume that it is not an unsurmountable task. In the following logical form that is meant to capture the reading (18b), it is rather unspecifically represented by the relation symbol $R$. (A standard characterization of an event consists of an event description with an event variable as its first argument and the thematic role fillers as further arguments, e.g. used $(e)\left(\operatorname{pen}_{x}\right)(\mathrm{j})$. Deviating from Moltmann's suggestion, I prefer to locate the event dependence of the modifiers in the determination of the comparison set. A notational reflex is the annotation of $X_{e}$ rather than having an additional event argument for the modifier.)

$$
\begin{aligned}
& \exists e: \operatorname{used}(e)\left(\operatorname{pen}_{x} \wedge \operatorname{ncw}\left(x, \mathbf{X}_{e}\right)\right)(\mathrm{j}) \\
& \text { where } \mathrm{X}_{e}=\left\{x: x \in \operatorname{PEN} \wedge \exists e_{i} \mathrm{R}\left(e, e_{i}\right) \wedge \operatorname{THEME}\left(x, e_{i}\right)\right\}
\end{aligned}
$$

The comparison class is a set defined by the individuals that are both a pen and the theme $e^{3}$ of some event of $e_{i}$ of the same type of $e$. What is crucial in the light of the following is that the comparison set may be entered into the scope of the existential event quantifier since it is part of a non-quantificational, weak NP. Thus, the event index of $\mathrm{X}_{e}$ is bound and the comparison set can indeed be determined with respect to the event description to which the modified NP contributes. Why does this not work for quantificational, strong NPs? The answer involves two related considerations why the NP as a whole cannot be properly interpreted on the background of a given context.

Assuming the standard treatment of quantificational NPs as quantifying into the scope domain of a (minimal) event, we would arrive at a logical form as follows (suppressing the definition of $X_{e}$ ).

$$
\forall x\left[\left[\operatorname{pen}_{x} \wedge \operatorname{new}\left(x, X_{e}\right)\right] \rightarrow \exists c \operatorname{used}(e)(x)(\mathrm{j})\right.
$$

Here, the problem is the unbound event variable of $X_{e}$. The comparison set has to be determined on the basis of some $e$ that, as a free variable, cannot be evaluated outside the the scope of the event description of the verb. ${ }^{4}$

If, on the other hand, we allow non-standardly for a logical form as in (19), we find that the domain presupposition of every new pen cannot be satisfied when the comparison set is to be defined with respect to the event.

$$
\exists e \forall x\left[\left[\operatorname{pen}_{x} \wedge \operatorname{new}\left(x, X_{e}\right)\right] \rightarrow \operatorname{used}(e)(x)(\mathrm{j})\right]
$$

The domain presupposition requires that $\left[\operatorname{pen}_{x} \wedge\right.$ new $\left.\left(x, X_{e}\right)\right]$ be determined with respect to the previous context. Conflictingly, the determination of the comparison set within the domain of the strong NP depends on the event described in the current sentence. Thus, the restriction new pen cannot be evaluated outside the scope of the event quantifier, contrary to what is required by its domain presupposition. Concluding this excursion on Moltmann's notion of domain presupposition, I return to the DE in comparatives.

There is an obvious parallel between event-dependent modifiers and the comparative adjective in attributive comparatives. Apart from being noun 
modifiers, both kinds of expressions relate an individual to some comparison instance that is defined either with respect to the sentence they occur in or on the basis of the previous context. Both the thing compared and the comparison instance have to be taken from a suitable comparison set. An obvious difference concerning the determination of the respective comparison instance and the comparison set is that, in attributive comparatives, it cannot be taken as dependent on the particular event description in which the comparative $\mathrm{NP}$ occurs. For instance, when $\mathrm{Al}$ asked for a bigger piece of cake (than any of those in the display case), it is not his request (the "ask-for"-event) that helps deciding what comparison set is meant. Moreover, the possibility for a complement provides us with a different device than event-dependency for determining elements, i.e. comparison instances, in a comparison set. In the following two sections, I provide the details for how the different factors in a comparative influence the identification of a comparison instance and a comparison set.

\section{Analysis of the comparative NP}

Consider the following example of an attributive comparative without complement:

(23) Sue had a piece of cake, and Al had a bigger piece of cake.

In section 2., I motivated that a comparative depends on a contextually given comparison instance from a comparison set. Let's preliminarily account for this by the following logical form for the comparative adjective.

(24) bigger $\Longrightarrow \lambda N \lambda x . N(x) \wedge x>_{\text {big }}$ a $\quad$ where $\mathbf{a}, x \in c[N]$

The comparative adjective is a noun modifier, i.e. a function that takes a noun denotation $N$ into a property of individuals. The property is characterized as being comparatively related to some contextually given individual a. The comparative relation $>_{\text {big }}$ may be part either of the lexical specification of a gradable adjective or of the comparative morpheme -er (cf. Klein (1991) for a discussion of different approaches). It will be defincd as a relation among clements of the sortal range of a gradable expression that satisfies the following axiom schemata (from (Hoeksema 1983)). The two axioms suffice to characterize each $>_{a}$ as a strict partial order.

(A1) $\forall x, y, z\left[x>_{\alpha} y \rightarrow\left[x>_{\alpha} z \vee z>_{C} y\right]\right]$

(almost-connectedness)

(A2) $\forall x, y\left[x>_{\alpha} y \rightarrow y \ngtr_{\alpha} x\right]$

(asymmetry)

In this paper, I deliberately avoid to refer to degrees, extents, standards or similar notions usually used in the analysis of comparative constructions. It docsn't seem to be necessary to postulate other types of entities than individuals (that may be collected into sets) for the analysis of plain comparatives. Plain means that the comparative is not further modified by degree modifiers 
as for instance much or three times in a much bigger piece or a three times faster car. It can be shown that, on the basis of the above axioms, an analysis of plain comparatives that makes use of, for instance, degrees is reducible to a corresponding analysis that doesn't (see Klein (1991) for details). An obvious practical consideration to avoid reference to degrees is that the logical forms will be much easier to read when we don't have to worry about degree predicates, degree quantifiers etc. In the following, I only mention degrees or standards when it seems to be commanded by specific constructions.

(24) adds the constraint on both the comparison instance a and the things compared that they be taken from a certain comparison class. In the case of attributive comparatives, I assume that the comparison class is determined by the noun interpretation. Consider the above example for illustration: it does not make sense to take into consideration the two salient individuals, namely Sue and the cake she had, when we compare the size of the piece of cake $\mathrm{Al}$ had. Now, somewhat unspecifically, the set $c[N]$ in (24) should be taken to be the set of things that are both $N$ and part of the current contextual universe. Probably this is too strong of a requirement for the determination of a comparison class as witnessed for instance by examples like (25), where the property of the thing compared and the property of the comparison instance are of a common kind, e.g. automotive means of transport.

(25) Sue owns a pretty fast motorbike, but $\mathrm{Al}$ owns a faster car.

Although it remains an open problem, how exactly a comparison set may be determined by a variety of contextual factors, I take it for granted that the interpretation of the noun in an attributive comparative contributes to its determination.

Let me return to the question of the comparison instance. It is not quite correct, to define the required comparison instance as a contextually salient individual in the sortal range of the comparative adjective. Often, we find several possible comparison instances. Take the following:

(26) Al didn't like any piece of cake on display. He ordered a bigger piece.

(26) tells us two things: (i) it is possible to have a set of comparison instances rather than just a single individual comparison instance; and (ii), the comparative relation is stated with respect to any element in this set of instances. Given that a single individual comparison instance can be viewed as a singleton set, we can revise the logical form in (24) as follows.

(27) faster $\Longrightarrow \lambda N \lambda x . N(x) \wedge \forall y \in \mathbf{X}: x>_{\text {fast }} y$

where $\mathrm{X} \subseteq c[N]$ and $x \in c[N]$

The universal quantification over the elements of $\mathbf{X}$ guarantees that the thing compared is in the comparative relation to each one of them. Since the comparison instance has been revised to be a set. $\mathbf{X}$, the constraint on $\mathbf{X}$ has to be revised accordingly: $\mathrm{X}$ has to be a subset of the comparison set $c[N]$. 
A minor revision has to be added in order to facilitate the composition when the comparative has a complement. In (28), the set constant $\mathbf{X}$ has been replaced by a set variable $X$ that ranges over some set of comparison instances. In case there is a complement, it will be the second argument of the comparative adjective.

(28) faster $\left.\Longrightarrow \lambda N \lambda X \lambda x . N(x) \wedge \forall y \in X: x>_{\text {fast }} y\right]$

where $X \subseteq c[N]$ and $x \in c[N]$

For the following, (28) will be our logical form for the comparative adjective. The composition of a complementless comparative NP is standard. First, the comparatives adjective combines with a noun via function application. In case there is no complement, the comparative NP's determiner combines with the resulting relation between the property of comparison instances and individuals via function composition. The logical form of an entire comparative clause without complement will then be an abstraction over properties of comparison instances, which has to be satisfied by a contextually given set. This concludes the discussion of the comparative NP. In the next section, I discuss the contribution of the complement to the determination of the comparison instance.

\section{Analysis of the complement}

I turn to hypotactic comparatives first. The main example will be the following.

(29) $\mathrm{Al}$ ate a bigger piece of cake than Sue a.te.

Let me start with a syntactic observation. It has been argued that there is syntactic evidence to treat the comparative complement similar to indirect questions. Among other syntactic evidences (see Klein (1991) for a summary and literature), it is noted by Chomsky (1977) that, in non-standard English, predicative comparative constructions may have the form of overt wh-constructions.

(30) I am taller than what you are.

German dialects lend further support to this obscrvation. Here, the complement may take the form of a how-question rather than a what-question. To witness, compare a litcrary example by Heinrich Heine (the fact that it is an equative rather than a comparative can be neglected; the phonomenon can be found in either construction).

(31) [...] deren Grossmut ist so gross als wie das Loch der Armenbüchs'.

'their generosity is as big as how the hole of the collecting box' 
I follow a proposal by Lerner and Pinkal (1995), who assume, gencralizing the observation for predicative comparatives, that the syntax of thancomplements involves an implicit wh-operator in both predicative and attributive comparatives. The different forms of wh-complements give rise to the question how exactly the wh-complement is to be analysed semantically, i.e. what semantics the implicit wh-operator ought to be assigned. There appear to be two possible options, illustrated by the following paraphrases of (29).

(32) Al ate a bigger piece of cake than what Sue did.

(33) $\mathrm{Al}$ atc a bigger piece of cake than how big pieces of cakc Sue did.

(32) seems to suggest that what the complement asks for is a set of things, while the complement in (33) takes the form of what one may classify as a degree question. Is there a way to account for the two options for whcomplements in a unified analysis? I think there is. Let me refer to an idea in (Heim 1987) concerning the analysis of specific-nonspecific ambiguities in what-questions. Heim argues that what-questions interpreted non-specifically can be analysed as involving a (narrow scope) occurrence of 'something of kind $x^{\prime}$. In analogy, we might interpret the what-paraphrase in (32) as involving an occurrence of 'something of kind $x$ ' and assume that the degree interrogative in (33) realizes a particular instantiation of the kind $x$ in question. Since the postulated wh-operator in the syntactic analysis of the comparative complement is an implicit operator, I think it is not necessary to insist on a particular instantiaton as long as it is not explicitly required by the question predicate (i.e. Sue did in (32) and (33)). An example as (34) certainly requires reference to something like degrees or standards.

(34) $\mathrm{Al}$ ate a sweeter piece of cake than (what) was good for his teeth.

However, let me stick to the policy of the previous section and continue to compare individuals rather than degrees or standards. Thus restricted, I follow the standard assumption that the implicit wh-operator scmantically introduces an abstraction over a free variable within the denotation of the question predicate, i.e. an abstraction over an individual variable. Then, the denotation of the entire comparative wh-complement will simply be a set of individuals. For instance, the denotation of the complement than (what) Al a.te is a set represented by the following logical form.

(35) $\lambda y \cdot a t e^{\prime}(a 1, y)$

A set of individuals is exactly what is needed as the second argument of a comparative adjective. Since we load all the responsibility for extablishing a particular comparative relation on the comparative adjective that, in addition, induces a constraint on a set of comparison instances, we don't have to worry about properties of the free variable implicitly contained in the information given by the question predicatc when none such property is predicated. 
Moreover, it is questionable whether implicit properties should be assumed at all when explicit occurences of such predications yield ungrammatical results:

(36) *Sue ate a bigger muffin than Al baked a big scone.

Relatedly, it has been argued (cf. Lerner and Pinkal (1995, p.7)) that the implicit noun in the complement is a one-pronoun that is subject to an anaphoric mechanism similar to One Anaphora. In contrast to the current proposal that the complement denotes a set, their proposal yields a proposition for the complement where the frec variable is existentially quantificd. This conclusion, however, is not supported by the German data they mention as cvidence.

(37) Sue besitzt einen schnelleren Wagen als Al einen hat.

'Sue owns a faster car than Al one has' (Lerner and Pinkal's (52))

(38) Sue besitzt einen schnelleren Wagen als $\mathrm{Al}$ welche hat.

'Sue owns a faster car than Al some has'

The one-pronoun in (37) is just a special instance of the more general plural pronoun in (38), which rather supports the view that a set of individuals is anaphorically related to some contextually salient property. Its resolution would be guaranteed under recourse to the constraint that it has to be a subset of the comparison set $c[N]$.

To summarize, I assume that the hypotactic complement consists of two parts, an implicit wh-operator and a question predicate. Taking the syntactic evidence seriously, I propose that the complement has the semantics of an indirect question. The assumption that the wh-operator is represented as an abstraction over individuals provides us with a respective set that can be identified as the set of comparison instances.

Providing the complement with a question semantics has a further advantage concerning the problem of quantified NPs and connectives occurring within the complement. Consider the following examples from (Lerner and Pinkal 1995).

(39) George owns a faster car than every policeman.

(40) George owns a faster car than Bill and Richard.

The problems arise with the readings on which George's car is compared, in (39), to the different cars owned by the different policemen and, in (40), to both Bill's cars and Richard's cars. The less accessible readings that involve a comparison to, respectively, the cars co-owned by the policemen and the cars co-owned by Bill and Richard are usually taken to be the unproblematic basic case. To account for the problematic readings, for instance, von Stechow (1984) refers to a non-standard scoping mechanism and Lerner and Pinkal (1995) stipulate a non-standard composition operation of generalized function application ${ }^{5}$. Apart from the fact that non-standard mechanisms have 
to be assumed, another weakness of these accounts that they analyse the preferred reading by a derived logical form. However, the already questionable "basic" reading with an every NP, seems to disappear when employing other quantifiers:

(41) George owns a faster car than most/few/the three policemen.

A reading in which George's car is said to be faster than the car(s) co-owned by most, few or the three policemen is very unlikely. For a solution of the problems within the current analysis of the semantics of the complement, we may appeal to the standard observation that quantifiers ocurring in the question predicate of indirect questions induce functional readings (cf. Groenendijk and Stokhof (1982) and Engdahl (1986)). Skipping the details of the exact composition of a functional wh-complement, its representation will be roughly as follows.

$$
\lambda f \forall x\left[\operatorname{policeman}^{\prime}(x) \rightarrow \text { own }^{\prime}(x, f(x))\right]
$$

Here, it is asked for a function $f$ such that each policeman owns something that is the value of $f$ with respect to himself. The denotation of $f(x)$ is a set of things owned by $x$. Thus, the denotation of (42) will be a set of set of things owned by the individual policemen. Each of the sets given by $f$ serves as a possible set of comparison instances which has to be a subset of the comparison set. The problem of connectives within complements is accounted for analogously.

I add two final remarks. The analysis of the hypotactic complement as an indirect question also nicely accounts for the fact that negative polarity item occurrences in the complement are licensed. Since questions generally are negative polarity environments with the respective monotonicity properties, I take this as further support for the indirect question analysis of the complement. See (Hoeksema 1983) for a discussion of the comparative as a negative polarity environment. Second, the characterization of the comparison instance as a set of possibly more than a single element accounts for Lerner and Pinkal's (1995) observation that the comparison instance is not anaphorically accessible from the discourse following the comparative sentence, since anaphora relate to an individual, not a set.

Let's turn to paratactic complements. An example for a comparative with a paratactic complement is (43).

(43) $\mathrm{Al}$ ordered a bigger piece of cake than the one/anyone on display.

In contrast to hypotactic comparatives where the complement is clausal, the complement of paratactic comparatives, on the surface, only consists of the complement marker than and a plain NP constituent. My proposal for the semantics of paratactic complements is actually quite simple. Since the comparative adjective requires a comparison instance or, more generally, a set of comparison instances, I take the complement constituent to denote the 
instance or, respectively, the set (as above, we might assume a general set forming mechanism for the complement). Support for this view can be drawn from the observation that individual denoting expression like proper names or singular definite descriptions and expressions denoting (maximal) sets like any-NPs or plural definite descriptions are preferred in paratactic complements while other NPs appear much less acceptable.

(44) ?? Al ordered a bigger piece of cake than three/most/few pieces.

As a consequence of the direct analysis for the paratactic complement, i.e. that the analysis does not assume an implicit wh-operator, we are deprived of the indirect question as a negative polarity environment. In this respect, there has been some dispute about any-NPs being negative polarity items (NPIs). I think, however, that we have good motivation for a free choice any analysis. The predicative comparative in (45) has been used as support for a NPI-any analysis (Klein 1991, ex.(127b)). It is claimed that both NPIs have to be licensed by the comparative as a negative environment.

(45) Sue is taller than any boy that I ever met.

However, observations about negative islands show that an NPI in a relative clause cannot be licensed by an operator outside the scope of the NP (see Ladusaw (1996) for an overview). I take it that ever is licenscd because it occurs in the restriction of a universal, i.c. the free choice any.

The analysis of paratactic complements may be somewhat cursory. A detailed examination of the different kinds of NPs is certainly desirable as would be a unified analysis for both paratactic and hypotactic comparatives. Such an analysis probably has to make reference to degrees or standards, which I decided to avoid. It has been shown that hypotactic comparatives semantically analysed as indirect questions proves useful in the explanation of a variety of problems. Furthermore, I take the above as good evidence that comparatives involve both a comparison instance, which may be a set, and a contextually available comparison set. I argued that they have to be kept apart. We exploit their characterization in the following.

\section{The analysis of the DE in comparatives}

Consider again our original observation about the DE in complementless comparatives.

(7) Of those cars, Sue bought one. George bought every faster car.

(8) Sue bought a car. \#George bought cvery faster car.

(9) Sue bought a car. George bought a faster car.

Exploiting the characterization of comparison instance and comparison set, the explanation of the contrast in acceptability between (7) and (8) runs as 
follows. The interpretation of every faster car in (7) depends on a comparison set $c\left[c a r^{\prime}\right]$ in its domain. Since the sentence preceding the comparative provides a context in which the contextually available cars are restricted to a specific set of cars, the domain presupposition of the strong comparative NP can be satisfied. The co-intersective quantifier ranges over elements of the definite comparison set rather than anything which is a car. Having identified the comparison instance, i.e. the car Sue bought, a truth value for the comparative sentence can be computed. Contrastingly in (8), there is no definite comparison set the comparison instance is taken from. The constraint that the comparison instance has to be a subset of some comparison set allows for a variety of instantiations. However, since the domain presupposition for strong quantifiers requires a definite domain, the comparative sentence is subject to a presupposition failure. For (9), it is only required that both Sue's car and George's car bc elements of some comparison set regardless of its exact definition. The indefinite comparative NP does not presuppose its domain and, thus, does not require a definite comparison set.

Regarding the DE in comparatives with complements, the above explanation in terms of presupposition failure applies analogously. If no definite comparison set can be found, this results in a presupposition failure when strong comparative NPs are involved in the comparative. However, there are some puzzling differences between paratactic and hypotactic complements which are not entirely clear to me yet. Consider the elliptical (46), which is ambiguous between the paraphrased paratactic and hypotactic resolution of the elliptical complement whereas (47) does not seem to give rise to the respective ambiguity. (I chose contestants as the noun of the comparative $\mathrm{NP}$ to suggest that there is a definite comparison set-the participants in a certain contest usually determine such a definite set.)

(46) Sue defeated a stronger contestant than Al.

a. 'Sue defeated a stronger contestant than how strong $\mathrm{Al}$ is.'

b. 'Sue defeated a stronger contestant than what contestants Al did.'

(47) Sue defeated every stronger contestant than Al.

a. 'Sue defeated every stronger contestant than how strong $\mathrm{Al}$ is.'

b: 'Sue defeated every stronger contestant than what contestantsAl did.'

Under the assumption that contestant provides a definite comparison set, no difference in acceptability between the paratactic reading of (47) and its hypotactic reading is expected according to the above explanation of the DE. Nevertheless, the hypotactic reading seems to be distinctly less admissible than the paratactic reading. An account for this difference may be found in the different semantics of the respective complements rather than being related to the DE. While the paratactic complement is always interpreted 
as definite (individual or set), the question semantics of the hypotactic complement yields an unspecific comparision instance. It may be our lack of knowledge about the comparison instance, that precludes the reading with a hypotactic complement. It cannot be decided who Sue defeated when it is not known who exactly $\mathrm{Al}$ defeated. There are no such limitations for the readings in (46) with a weak comparative NP.

Another issue regarding complemented comparatives that I have to leave to further investigation concerns accommodation of the comparison set. Let me just give a rough idea of what is at stake. Note that (48) is not as hopeless as (49).

(48) Al ate the bigger piece of cakc than Sue.

(49) ${ }^{\#} \mathrm{Al}$ ate the bigger piece of cake than every student.

Suppose that everyone had just one piece of cake. In (48) it is possible to accommodate a comparison set of exactly two pieces of cake consisting of the one eaten by $\mathrm{Al}$ and the other eaten by Sue. With this comparison set at our disposition, the uniqueness condition imposed by the definite comparative NP is satisfied. For (49), it is not possible to accommodate a definite comparison set in a similar fashion. Since the complement provides us with a range of possible comparison instances, i.e. a single piece or a set of pieces for each student, there would be a range of possible comparison sets. Each one of those would contain the piece of cake eaten by $\mathrm{Al}$ and the one eaten by some student.

\section{A note on comparative ellipsis}

The analysis of the complement in section 5. confines me to a particular view about the resolution of comparative ellipsis. First, paratactic complements cannot be an instance of comparative ellipsis. Since the interpretation of the constituent within the paratactic complement directly establishes the comparison instance, no resolution of any additional information is required. Regarding hypotactic: complements, I need an account of how the question predicate may be completed, i.e. how the missing information may be retrieved from the previous context. On the surface, an elliptical complement may appear in two different guises. VP ellipsis contains an overt occurrence of an auxiliary or modal verb. Bare remnant ellipsis only involves one or more constituent occurrences without a verb. I follow a widely accepted assumption that resolution takes place on some level of logical form. Furthermore, I assume that some sort of anaphoric mechanism is responsible for ellipsis resolution. The auxiliary in VP cllipsis supplies an overt anaphoric element whereas an implicit one is demanded in bare remnant ellipsis. The logical for $m$ of the complement contains an implicit relation that is anaphorically related to an anteccdent. The arity of the implicit relation will be fully determined by the overt material in the ellipsis together with the requirement of a free 
variable in the question predicate to be bound by the implicit wh-operator. Necessitated by the question semantics of the complement, the relation is at least unary. An example for a missing property is the following bare remnant complement, where the resolved question predicate may be paraphrased as what Sue had yesterday.

(j0) Sue had a bigger piece of cake than yestcrday.

The assumption of an anaphoric relation with flexible arity avoids the problems of an analysis of elliptical comparatives as some sort of antecedent contained deletion. Specifically, it cannot be motivated that the resolution of e.g. (50) forces scoping of the comparative NP as predicted in main stream analyses of antecedent contained deletion (cf. Lerner and Pinkal (1995) for further details). I argued elsewhere (Beil 1996) that antecedent contained deletion can also be analysed as involving a flexible relation.

\section{Open ends}

Since the paper aimed at an explanation of the DE in comparatives, the attention was restricted to attributive comparatives in their various forms. This restriction motivated the somewhat problematic decision to relate only individuals in a comparative relation. I think, though, this decision does not diminish the results concerning the $\mathrm{DE}$. Of course, it remains to be shown how the analysis can be embedded in an account for comparatives in general. A generalization of the account for the hypotactic complement analysed as an indirect question may prove useful here. To my knowledge, the indirect question analysis of comparative complements has not been investigated from a semantic point of view. I mentioned that the analysis may show more merits with respect to further semantic problems posed by the complement. (i) The complement is a negative polarity environment. (ii) The comparison instance is anaphorically inaccessible. In addition, (iii) a clausal negation in the complement is anomalous (Sue bought a faster car than Al didn't).

Hopefully, further research will yield positive results.

\section{Endnotes}

* I am grateful to Cleo Condoravdi, Tim Fernando, Antje Rossdeutscher and Carl Vogel for their help.

1 I call the thing or things which the item of comparison is compared to the comparison instance. In a comparative clause with complement, the comparison instance is given with the complement.

2 Since I will not be concerned in the following with specific problems related to presupposition projection behaviour of complex determiners, I confine myself to Moltmann's informal characterization of domain presupposition and refer the reader to her paper for the formalization.

3 A more cautious formulation might be preferred: let the comparison class 
be a sct of individuals that participate in an event of the same type; the elements do not necessarily have to be assigned to the same thematic role as in the original event.

4 I think that Moltmann's explanation stops short here. The problem is not that the free variable cannot be evaluated but that a given context may provide several possible specifications of $e$ via different variable assignment functions. (In dynamic semantics, a context is often characterized as a set of assignments.) This results in a different comparison set for different specifications. Because of that, the domain presupposition cannot be satisfied.

5 Although Lerner and Pinkal (1995) argue that their operation is logically harmless since it can be defined in terms of plain function application, I conjecture that there are serious problems if one allows for unrestricted application in the composition. For instance, its application in the composition of a subject NP and a transitive verb will have fatal results. Moreover, it is not shown that the operation is of use for other constructions than for comparatives.

\section{References}

Beil, F.: 1996, On antecedent contained ellipsis. to appear in Proceedings of the Colloque de Syntaxe et Semantique de Paris, October 12-14, 1995.

Chomsky, N.: 1977, On wh movement, in P. W. Culicover, T. Wasow and A. Akmajian (eds), Formal Syntax, Academic Press, New York.

Engdahl, E.: 1986, Constituent Questions, Reidel, Dordrecht.

Groencndijk, J. and Stokhof, M.: 1982, Semantic analysis of wh-complements, Linguistics and Philosophy 5, 175-233.

Heim, I.: 1987, Where does the definiteness restriction apply? Evidence from the definiteness of variables, in E. J. Reuland and A. G. B. ter Meulen (eds), The Representation of (In)definiteness, Vol. 14 of Current Studies in Linguistics Series, MIT Press, Cambridge/MA, chapter 2.

Higginbotham, J.: 1987, Indefiniteness and predication, in E. J. Reuland and A. G. B. ter Meulen (eds), The Representation of (In) definiteness, Vol. 14 of Current Studies in Linguistics Series, MIT Press, Cambridge/MA, chapter 3 .

Hoeksema, J.: 1983, Negative polarity and the comparative, Natural Language and Linguistic Theory 1(3), 403-434.

Keenan, E. L.: 1996, The semantics of determiners, in S. Lappin (ed.), The Handbook of Contemporary Semantic Theory. Blackwell, ()xford, pp. 4164.

Klein, E.: 1980, A semantics for positive and comparative adjectives, Linguistics and Philosophy 4, 1-45. 
Klein, E.: 1991, Comparatives, in A. von Stechow and D. Wunderlich (eds), Semantik. Ein internationales Handbuch der zeitgenössischen Forschung, de Gruyter, Berlin, chapter 32, pp. 673-691.

Ladusaw, W. A.: 1996, Negation and polarity items, in S. Lappin (ed.), The Handbook of Contemporary Semantic Theory, Blackwell, Oxford, pp. 321-342.

Lerner, J.-Y. and Pinkal, M.: 1995, Comparative ellipsis and variable binding, Proceedings of SALT V, Cornell University, CLC Publications.

* http://xxx.lanl.gov/ps/cmp-lg/9507005

Moltmann, F.: 1996, Domain-related dynamic semantics and the weak-strong distinction among quantifiers. Ms., CUNY, Dept. of Philosophy.

von Stechow, A.: 1984, Comparing semantic theories of comparison, Journal of Semantics 3, 1-77. 